\title{
Stability Analysis of Three-Species Almost Periodic Competition Models with Grazing Rates and Diffusions
}

\author{
Chang-you Wang, ${ }^{1,2}$ Rui-fang Wang, ${ }^{2,3}$ Ming Yi, ${ }^{4}$ and Rui $\mathrm{Li}^{2,3}$ \\ ${ }^{1}$ Institute of Applied Mathematics, Chongqing University of Posts and Telecommunications, \\ Chongqing 400065, China \\ ${ }^{2}$ Key Laboratory of Network control \& Intelligent Instrument, Chongqing University of \\ Posts and Telecommunications, Ministry of Education, Chongqing 400065, China \\ ${ }^{3}$ Automation Institute, Chongqing University of Posts and Telecommunications, Chongqing 400065, China \\ ${ }^{4}$ College of Computer Science and Technology, Chongqing University of Posts and Telecommunications, \\ Chongqing 400065, China
}

Correspondence should be addressed to Chang-you Wang, wangcy@cqupt.edu.cn

Received 10 May 2011; Accepted 2 June 2011

Academic Editor: Zhengqiu Zhang

Copyright (c) 2011 Chang-you Wang et al. This is an open access article distributed under the Creative Commons Attribution License, which permits unrestricted use, distribution, and reproduction in any medium, provided the original work is properly cited.

\begin{abstract}
Almost periodic solution of a three-species competition system with grazing rates and diffusions is investigated. By using the method of upper and lower solutions and Schauder fixed point theorem as well as Lyapunov stability theory, we give sufficient conditions to ensure the existence and globally asymptotically stable for the strictly positive space homogenous almost periodic solution, which extend and include corresponding results obtained by Q. C. Lin (1999), F. D. Chen and X. X. Chen (2003), Y. Q. Liu, S. L, Xie, and Z. D. Xie (1996).
\end{abstract}

\section{Introduction}

In this paper, we study the following three-species competition system with grazing rates and diffusions:

$$
\begin{aligned}
& \frac{\partial v_{1}(x, t)}{\partial t}=k_{1}(t) \Delta v_{1}(x, t)+v_{1}(x, t)\left[a_{1}(t)-b_{1}(t) v_{1}(x, t)-c_{1}(t) v_{2}(x, t)-d_{1}(t) v_{3}(x, t)\right]+f_{1}(t), \\
& \frac{\partial v_{2}(x, t)}{\partial t}=k_{2}(t) \Delta v_{2}(x, t)+v_{2}(x, t)\left[a_{2}(t)-b_{2}(t) v_{1}(x, t)-c_{2}(t) v_{2}(x, t)-d_{2}(t) v_{3}(x, t)\right]+f_{2}(t), \\
& \frac{\partial v_{3}(x, t)}{\partial t}=k_{3}(t) \Delta v_{3}(x, t)+v_{3}(x, t)\left[a_{3}(t)-b_{3}(t) v_{1}(x, t)-c_{3}(t) v_{2}(x, t)-d_{3}(t) v_{3}(x, t)\right]+f_{3}(t),
\end{aligned}
$$


where $(x, t) \in \Omega \times R^{+}, \Omega \subseteq R^{n}$ is the bounded open subset of $R^{n}$ with smooth boundary $\partial \Omega$, which represent the habitat domain for three species. System (1.1) is supplement with boundary conditions and initial conditions:

$$
\begin{gathered}
\frac{\partial v_{i}(x, t)}{\partial n}=0, \quad i=1,2,3,(x, t) \in \partial \Omega \times R^{+}, \\
v_{i}(x, 0)=v_{i 0}(x) \geq 0, \quad v_{i 0}(x) \not \equiv 0, i=1,2,3, x \in \bar{\Omega}
\end{gathered}
$$

where $\partial / \partial n$ denotes the outward normal derivation on $\partial \Omega$, and $v_{i}(x, t)$ represent the density of $i$ th species at point $x=\left(x_{1}, \ldots, x_{n}\right)$ and the time of $t$. Here, $k_{i}(t), a_{i}(t), b_{i}(t)$, $c_{i}(t), d_{i}(t)$, and $f_{i}(t)(i=1,2,3)$ denote the diffusivity rates, competition rates, and grazing rates, respectively. They are almost periodic functions in real number field $R$. $\Delta$ is a Laplace operator on $\Omega$.

System (1.1)-(1.3) describes the interaction among three species and is an important model in biomathcmatics, which has been intensively investigated, and much attention is carried to the problem [1-8]. When there is no diffusion, Jiang [1] and Lin [2] studied the existence, uniqueness, and stability on periodic solution and almost periodic solution for two-species competition system under the condition that the coefficients are the periodic function and almost periodic function, respectively; F. D. Chen and X. X. Chen [3] extended the results in [2] to n-species case. When there are no diffusion and grazing rates, Zhang and Wang $[4,5]$ investigated the existence of a positive periodic solution for a two-species nonautonomous competition Lotka-Volterra patch system with time delay and the existence of multiple positive periodic solutions for a generalized delayed population model with exploited term by using the continuation theorem of coincidence degree theory; $\mathrm{Hu}$ and Zhang [6] established criteria for the existence of at least four positive periodic solutions for a discrete time-delayed predator-prey system with nonmonotonic functional response and harvesting by employing the continuation theorem of coincidence degree theory. When there are no grazing rates, Pao and Wang [7] proved the stability for invariable coefficient case by utilizing the method of upper and lower solutions. Liu et al. [8] showed the stability on the periodic solution for $\mathrm{n}$-species competition system with grazing rates and diffusions. Nevertheless, generally speaking, the system does not always change strictly according to periodic laws, sometimes it changes according to almost periodic laws, and it is important to survey almost periodic solution for the multispecies competition system with grazing rates and diffusions. To sum up, we pay more attention to almost periodic solution of a threespecies competition system (1.1)-(1.3) with grazing rates and diffusions; in this paper, by using the method of upper and lower solutions and Schauder fixed point theorem as well as Lyapunov stability theory, we obtain sufficient conditions which ensure the existence and globally asymptotically stable for the strictly positive space homogenous almost periodic solution, which extend and include corresponding results obtained in $[2,3,8]$. Many other results on the periodic solution and almost periodic solution can be found in [9-16].

\section{Preliminary}

Firstly, we give out some definitions and lemmas.

Definition 2.1. Suppose that $f(t)$ is a continuous function in $R$. Then $f(t)$ is said to be almost periodic in $t \in R$ if for every $\varepsilon>0$ corresponds $T(\varepsilon)>0$ such that for any interval $I$ whose length is equal to $T(\varepsilon)$ there is at least one $\tau \in I$ such that

$$
|f(t+\tau)-f(t)| \leq \varepsilon, \quad \forall t \in R .
$$


Definition 2.2. If a smooth function $V(t)=\left(v_{1}(t), v_{2}(t), v_{3}(t)\right)$ satisfies (1.1) in $R^{+}$, and every component of $V(t)$ is the almost periodic function, we called that $V(t)$ is a spatial homogeneity almost periodic solution for (1.1), which is denoted by $V(t, T(\varepsilon))$.

Definition 2.3. For any nonnegative smooth initial data

$$
V(x, 0)=\left(v_{1}(x, 0), v_{2}(x, 0), v_{3}(x, 0)\right)=\left(v_{10}(x), v_{20}(x), v_{30}(x)\right) \geq 0, \quad V(x, 0) \not \equiv 0, x \in \Omega,
$$

if there exists a unique positive solution $V(x, t)=\left(v_{1}(x, t), v_{2}(x, t), v_{3}(x, t)\right)$ for the system (1.1) with boundary conditions (1.2), and $\lim _{t \rightarrow \infty}\left(V_{i}(x, t)-V_{i}(t, T(\varepsilon))\right)=0, i=1,2,3$, uniformly for $x \in \bar{\Omega}$, we called that spatial homogeneity almost periodic solution $V(t, T(\varepsilon))$ is globally asymptotically stable.

Definition 2.4. Suppose that $\bar{V}(x, t) \equiv\left(\bar{v}_{1}(x, t), \bar{v}_{2}(x, t), \bar{v}_{3}(x, t)\right), \underline{V}(x, t) \equiv\left(\underline{v}_{1}(x, t), \underline{v}_{2}(x, t)\right.$, $\left.\underline{v}_{3}(x, t)\right)$; if $\bar{V}(x, t) \geq \underline{V}(x, t)$ and

$$
\begin{aligned}
& \frac{\partial \bar{v}_{1}(x, t)}{\partial t} \geq k_{1}(t) \Delta \bar{v}_{1}(x, t)+\bar{v}_{1}(x, t)\left[a_{1}(t)-b_{1}(t) \bar{v}_{1}(x, t)-c_{1}(t) \underline{v}_{2}(x, t)-d_{1}(t) \underline{v}_{3}(x, t)\right] \\
& +f_{1}(t), \quad(x, t) \in \Omega \times R^{+}, \\
& \frac{\partial \bar{v}_{2}(x, t)}{\partial t} \geq k_{2}(t) \Delta \bar{v}_{2}(x, t)+\bar{v}_{2}(x, t)\left[a_{2}(t)-b_{2}(t) \underline{v}_{1}(x, t)-c_{2}(t) \bar{v}_{2}(x, t)-d_{2}(t) \underline{v}_{3}(x, t)\right] \\
& +f_{2}(t), \quad(x, t) \in \Omega \times R^{+}, \\
& \frac{\partial \bar{v}_{3}(x, t)}{\partial t} \geq k_{3}(t) \Delta \bar{v}_{3}(x, t)+\bar{v}_{3}(x, t)\left[a_{3}(t)-b_{3}(t) \underline{v}_{1}(x, t)-c_{3}(t) \underline{v}_{2}(x, t)-d_{3}(t) \bar{v}_{3}(x, t)\right] \\
& +f_{3}(t), \quad(x, t) \in \Omega \times R^{+}, \\
& \frac{\partial \bar{v}_{i}(x, t)}{\partial n} \geq 0, \quad i=1,2,3,(x, t) \in \partial \Omega \times R^{+}, \\
& \bar{v}_{i}(x, 0) \geq v_{i 0}(x), \quad i=1,2,3, x \in \bar{\Omega}, \\
& \frac{\partial \underline{v}_{1}(x, t)}{\partial t} \leq k_{1}(t) \Delta \underline{v}_{1}(x, t)+\underline{v}_{1}(x, t)\left[a_{1}(t)-b_{1}(t) \underline{v}_{1}(x, t)-c_{1}(t) \bar{v}_{2}(x, t)-d_{1}(t) \bar{v}_{3}(x, t)\right] \\
& +f_{1}(t), \quad(x, t) \in \Omega \times R^{+}, \\
& \frac{\partial \underline{v}_{2}(x, t)}{\partial t} \leq k_{2}(t) \Delta \underline{v}_{2}(x, t)+\underline{v}_{2}(x, t)\left[a_{2}(t)-b_{2}(t) \bar{v}_{1}(x, t)-c_{2}(t) \underline{v}_{2}(x, t)-d_{2}(t) \bar{v}_{3}(x, t)\right] \\
& +f_{2}(t), \quad(x, t) \in \Omega \times R^{+}, \\
& \frac{\partial \underline{v}_{3}(x, t)}{\partial t} \leq k_{3}(t) \Delta \underline{v}_{3}(x, t)+\underline{v}_{3}(x, t)\left[a_{3}(t)-b_{3}(t) \bar{v}_{1}(x, t)-c_{3}(t) \bar{v}_{2}(x, t)-d_{3}(t) \underline{v}_{3}(x, t)\right] \\
& +f_{3}(t), \quad(x, t) \in \Omega \times R^{+}, \\
& \frac{\partial \underline{v}_{i}(x, t)}{\partial n} \leq 0, \quad i=1,2,3,(x, t) \in \partial \Omega \times R^{+}, \\
& \underline{v}_{i}(x, 0) \leq v_{i 0}(x), \quad i=1,2,3, x \in \bar{\Omega},
\end{aligned}
$$

we called $\bar{V}(x, t)$ and $\underline{V}(x, t)$ a pair of ordered upper and lower solutions for systems (1.1)(1.3). 
Lemma 2.5 (see $[12,17])$. Suppose that $\bar{V}(x, t)$ and $\underline{V}(x, t)$ are a pair of ordered upper and lower solution for systems (1.1)-(1.3), then there exists a unique solution $V(x, t)$ for systems (1.1)-(1.3). Moreover, one has

$$
\bar{V}(x, t) \geq V(x, t) \geq \underline{V}(x, t) .
$$

For the almost periodic function $F(t)$ in $R$, one denotes $\widetilde{F}=\sup \{F(t), t \in R\}, \underset{\sim}{F}=$ $\inf \{F(t), t \in R\}$, and $M[F]=\lim _{(t-s) \rightarrow \infty}\left\{\int_{s}^{t} F(\tau) d \tau /(t-s)\right\}$. When $F(t)$ is $T$-periodic function, one denotes $M[F]=\int_{0}^{T} F(s) d s / T$.

\section{Main Results and Proofs}

Now we are in a position to state our main results and give our proofs.

Theorem 3.1. If $\underset{\sim i}{a} \underset{\sim i}{b} \underset{\sim}{b}, \underset{\sim}{d}, \underset{\sim}{d} \underset{\sim i}{f}$ are positive numbers, and

$$
\frac{\left(\tilde{b}_{i}+\tilde{c}_{i}+\tilde{d}_{i}\right)}{\underset{\sim i}{a}} \leq L=\min \left\{\sqrt{\frac{\underset{\sim}{b}}{\tilde{f}_{1}}}, \sqrt{\frac{\underset{\sim}{c}}{\tilde{f}_{2}}}, \sqrt{\frac{\underset{\sim 3}{d}}{\tilde{f}_{3}}}, \frac{(\underset{\sim 1}{d}+\underset{\sim 1}{c})}{\tilde{a}_{1}}, \frac{(\underset{\sim 2}{b}+\underset{\sim 2}{d})}{\tilde{a}_{2}}, \frac{(\underset{\sim 3}{b}+\underset{\sim 3}{b})}{\tilde{a}_{3}}\right\}
$$

are satisfied for $i=1,2,3$, then there exists a strictly positive spatial homogeneity almost periodic solution $V(t)=\left(\widehat{v}_{1}(t), \widehat{v}_{2}(t), \widehat{v}_{3}(t)\right)$ for $(1.1)$.

Proof. By the conditions in Theorem 3.1, we have

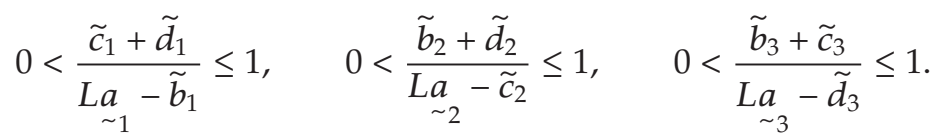

Let

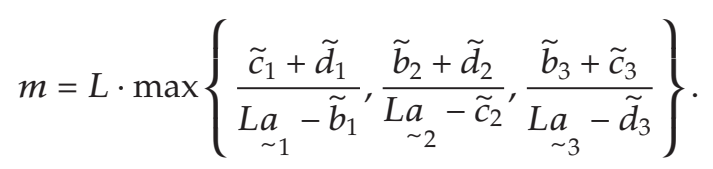

Then we have $0<m \leq L$, and

$$
\left(\tilde{c}_{1}+\tilde{d}_{1}\right) \frac{L}{m} \leq \underset{\sim 1}{\operatorname{ar}}-\tilde{b}_{1}, \quad\left(\tilde{b}_{2}+\tilde{d}_{2}\right) \frac{L}{m} \leq \underset{\sim 2}{\operatorname{ar}}-\tilde{c}_{2}, \quad\left(\tilde{b}_{3}+\tilde{c}_{3}\right) \frac{L}{m} \leq \underset{\sim 3}{\operatorname{ar}}-\tilde{d}_{3} .
$$

Therefore

$$
\begin{gathered}
\tilde{b}_{1}+\left(\tilde{c}_{1}+\tilde{d}_{1}\right) \frac{L}{m}-\underset{\sim 1}{f} m^{2} \leq \underset{\sim 1}{\operatorname{La}}, \quad \tilde{c}_{2}+\left(\tilde{b}_{2}+\tilde{d}_{2}\right) \frac{L}{m}-\underset{\sim 2}{f} m^{2} \leq \underset{\sim 2}{L a}, \\
\tilde{d}_{3}+\left(\tilde{b}_{3}+\tilde{c}_{3}\right) \frac{L}{m}-\underset{\sim 3}{f} m^{2} \leq \underset{\sim 3}{L a} .
\end{gathered}
$$


Furthermore, by the given conditions in Theorem 3.1, one has

$$
\begin{gathered}
\underset{\sim 1}{b}-\tilde{f}_{1} L^{2} \geq 0, \quad \underset{\sim 2}{c}-\tilde{f}_{2} L^{2} \geq 0, \quad \underset{\sim 3}{d}-\tilde{f}_{3} L^{2} \geq 0, \quad(\underset{\sim 1}{c}+\underset{\sim 1}{d}) \frac{m}{L} \geq m \tilde{a}_{1}, \\
(\underset{\sim 2}{b}+\underset{\sim 2}{d}) \frac{m}{L} \geq m \tilde{a}_{2}, \quad(\underset{\sim 3}{b}+\underset{\sim 3}{c}) \frac{m}{L} \geq m \tilde{a}_{3} .
\end{gathered}
$$

Thus

$$
\begin{gathered}
\underset{\sim 1}{b}+(\underset{\sim 1}{c}+\underset{\sim 1}{d}) \frac{m}{L}-\tilde{f}_{1} L^{2} \geq m \tilde{a}_{1}, \quad \underset{\sim 2}{c}+(\underset{\sim 2}{b}+\underset{\sim 2}{d}) \frac{m}{L}-\tilde{f}_{2} L^{2} \geq m \tilde{a}_{2,} \\
\underset{\sim 3}{d}+(\underset{\sim 3}{b}+\underset{\sim 3}{c}) \frac{m}{L}-\tilde{f}_{3} L^{2} \geq m \tilde{a}_{3} .
\end{gathered}
$$

Combining (3.5) and (3.7), we have

$$
\begin{array}{ll}
\tilde{b}_{1}+\left(\tilde{c}_{1}+\tilde{d}_{1}\right) \frac{L}{m}-\underset{\sim 1}{f} m^{2} \leq \underset{\sim 1}{L a}, \quad \underset{\sim 1}{b}+(\underset{\sim 1}{c}+\underset{\sim 1}{d}) \frac{m}{L}-\tilde{f}_{1} L^{2} \geq m \tilde{a}_{1}, \\
\tilde{c}_{2}+\left(\tilde{b}_{2}+\tilde{d}_{2}\right) \frac{L}{m}-\underset{\sim 2}{f} m^{2} \leq \underset{\sim 2}{\operatorname{La}}, \quad \underset{\sim 2}{c}+(\underset{\sim 2}{b}+\underset{\sim 2}{d}) \frac{m}{L}-\tilde{f}_{2} L^{2} \geq m \tilde{a}_{2}, \\
\tilde{d}_{3}+\left(\tilde{b}_{3}+\tilde{c}_{3}\right) \frac{L}{m}-\underset{\sim 3}{f} m^{2} \leq \underset{\sim 3}{L a}, \quad \underset{\sim 3}{d}+(\underset{\sim 3}{b}+\underset{\sim 3}{c}) \frac{m}{L}-\tilde{f}_{3} L^{2} \geq m \tilde{a}_{3} .
\end{array}
$$

Let

$$
H_{L}^{m}=\{(\phi(t), \varphi(t), \gamma(t)): \phi, \varphi, \gamma \text { are almost periodic in } t \in R, 0<m \leq \phi, \varphi, \gamma \leq L\}
$$

We consider the following system corresponding to (1.1):

$$
\begin{array}{ll}
\dot{v}_{1}=v_{1}\left(a_{1}(t)-b_{1}(t) v_{1}-c_{1}(t) v_{2}-d_{1}(t) v_{3}\right)+f_{1}(t), & t \in R^{+}, \\
\dot{v}_{2}=v_{2}\left(a_{2}(t)-b_{2}(t) v_{1}-c_{2}(t) v_{2}-d_{2}(t) v_{3}\right)+f_{2}(t), & t \in R^{+}, \\
\dot{v}_{3}=v_{3}\left(a_{3}(t)-b_{3}(t) v_{1}-c_{3}(t) v_{2}-d_{3}(t) v_{3}\right)+f_{3}(t), & t \in R^{+} .
\end{array}
$$

Let $z_{i}=1 / v_{i}, i=1,2,3$; then (3.10) becomes

$$
\begin{aligned}
& \dot{z}_{1}=b_{1}(t)-a_{1}(t) z_{1}+c_{1}(t) \frac{z_{1}}{z_{2}}+d_{1}(t) \frac{z_{1}}{z_{3}}-f_{2}(t) z_{1}^{2}, \\
& \dot{z}_{2}=c_{2}(t)-a_{2}(t) z_{2}+b_{2}(t) \frac{z_{2}}{z_{1}}+d_{2}(t) \frac{z_{2}}{z_{3}}-f_{2}(t) z_{2}^{2}, \\
& \dot{z}_{3}=d_{3}(t)-a_{3}(t) z_{3}+b_{3}(t) \frac{z_{3}}{z_{1}}+c_{3}(t) \frac{z_{3}}{z_{2}}-f_{3}(t) z_{3}^{2} .
\end{aligned}
$$


For any $(\phi(t), \varphi(t), \gamma(t)) \in H_{L}^{m}$, by $M\left[b_{1}\right]>0, M\left[c_{2}\right]>0, M\left[d_{3}\right]>0$, we observe [18] that

$$
\begin{aligned}
& \dot{z}_{1}=b_{1}(t)-a_{1}(t) z_{1}+c_{1}(t) \frac{\phi(t)}{\varphi(t)}+d_{1}(t) \frac{\phi(t)}{\gamma(t)}-f_{1}(t) \phi^{2}(t), \\
& \dot{z}_{2}=c_{2}(t)-a_{2}(t) z_{2}+b_{2}(t) \frac{\varphi(t)}{\phi(t)}+d_{2}(t) \frac{\varphi(t)}{\gamma(t)}-f_{2}(t) \varphi^{2}(t), \\
& \dot{z}_{3}=d_{3}(t)-a_{3}(t) z_{3}+b_{3}(t) \frac{\gamma(t)}{\phi(t)}+c_{3}(t) \frac{\gamma(t)}{\varphi(t)}-f_{3}(t) \gamma^{2}(t),
\end{aligned}
$$

have almost periodic solution:

$$
\begin{aligned}
& \widehat{z}_{1}(t)=\int_{-\infty}^{t} e^{-\int_{s}^{t} a_{1}(r) d r}\left[b_{1}(s)+c_{1}(s) \frac{\phi(s)}{\varphi(s)}+d_{1}(s) \frac{\phi(s)}{\gamma(s)}-f_{1}(s) \phi^{2}(s)\right] d s, \\
& \widehat{z}_{2}(t)=\int_{-\infty}^{t} e^{-\int_{s}^{t} a_{2}(r) d r}\left[c_{2}(s)+b_{2}(s) \frac{\varphi(s)}{\phi(s)}+d_{2}(s) \frac{\varphi(s)}{\gamma(s)}-f_{2}(s) \varphi^{2}(s)\right] d s, \\
& \widehat{z}_{3}(t)=\int_{-\infty}^{t} e^{-\int_{s}^{t} a_{2}(r) d r}\left[d_{3}(s)+b_{3}(s) \frac{\gamma(s)}{\phi(s)}+c_{3}(s) \frac{\gamma(s)}{\varphi(s)}-f_{3}(s) \gamma^{2}(s)\right] d s .
\end{aligned}
$$

By the system (3.13), we define a mapping $A$ :

$$
A(\phi(t), \varphi(t), \gamma(t))=\left(\widehat{z}_{1}(t), \widehat{z}_{2}(t), \widehat{z}_{3}(t)\right), \quad \forall(\phi(t), \varphi(t), \gamma(t)) \in H_{L}^{m}
$$

Combining (3.8) and (3.13), we have

$$
\begin{aligned}
& \widehat{z}_{1}(t) \geq \int_{-\infty}^{t} e^{-\tilde{a}_{1}(t-s)}\left[\underset{\sim 1}{b}+(\underset{\sim 1}{c}+\underset{\sim 1}{d}) \frac{m}{L}-\tilde{f}_{1} L^{2}\right] d s=\frac{1}{\tilde{a}_{1}}\left[\underset{\sim 1}{b}+(\underset{\sim 1}{c}+\underset{\sim 1}{d}) \frac{m}{L}-\tilde{f}_{1} L^{2}\right] \geq m, \\
& \widehat{z}_{1}(t) \leq \int_{-\infty}^{t} e^{-\underset{\sim 1}{a}(t-s)}\left[\tilde{b}_{1}+\left(\widetilde{c}_{1}+\tilde{d}_{1}\right) \frac{L}{m}-\underset{\sim 1}{f} m^{2}\right] d s=\underset{\sim 1}{\frac{1}{a}}\left[\tilde{b}_{1}+\left(\widetilde{c}_{1}+\tilde{d}_{1}\right) \frac{L}{m}-\underset{\sim 1}{f} m^{2}\right] \leq L, \\
& \widehat{z}_{2}(t) \geq \int_{-\infty}^{t} e^{-\tilde{a}_{2}(t-s)}\left[\underset{\sim 2}{c}+(\underset{\sim 2}{b}+\underset{\sim 2}{d}) \frac{m}{L}-\tilde{f}_{2} L^{2}\right] d s=\frac{1}{\tilde{a}_{2}}\left[\underset{\sim 2}{c}+(\underset{\sim 2}{b}+\underset{\sim 2}{d}) \frac{m}{L}-\tilde{f}_{2} L^{2}\right] \geq m, \\
& \widehat{z}_{2}(t) \leq \int_{-\infty}^{t} e^{-a(t-s)}\left[\widetilde{c}_{2}+\left(\tilde{b}_{2}+\tilde{d}_{2}\right) \frac{L}{m}-\underset{\sim 2}{f} m^{2}\right] d s=\frac{1}{a}\left[\widetilde{c}_{\sim 2}+\left(\tilde{b}_{2}+\tilde{d}_{2}\right) \frac{L}{m}-\underset{\sim 2}{f} m^{2}\right] \leq L, \\
& \widehat{z}_{3}(t) \geq \int_{-\infty}^{t} e^{-\tilde{a}_{3}(t-s)}\left[\underset{\sim 3}{d}+(\underset{\sim 3}{b}+\underset{\sim 3}{c}) \frac{m}{L}-\tilde{f}_{3} L^{2}\right] d s=\frac{1}{\tilde{a}_{3}}\left[\underset{\sim 3}{d}+(\underset{\sim 3}{b}+\underset{\sim 3}{c}) \frac{m}{L}-\tilde{f}_{3} L^{2}\right] \geq m, \\
& \widehat{z}_{3}(t) \leq \int_{-\infty}^{t} e^{-a(t-s)}\left[\tilde{d}_{3}+\left(\tilde{b}_{3}+\tilde{c}_{3}\right) \frac{L}{m}-\underset{\sim 3}{f} m^{2}\right] d s=\underset{\sim 3}{\frac{1}{a}}\left[\tilde{d}_{3}+\left(\tilde{b}_{3}+\tilde{c}_{3}\right) \frac{L}{m}-\underset{\sim 3}{f} m^{2}\right] \leq L .
\end{aligned}
$$


Therefore, $\left(\widehat{z}_{1}(t), \widehat{z}_{2}(t), \widehat{z}_{3}(t)\right) \in H_{L}^{m}$, that is, $A H_{L}^{m} \subset H_{L}^{m}$. If $A$ is uniformly boundness and equicontinuous, by Ascoli-Arzela theorem, $A$ is compact mapping.

It is obvious to obtain uniformly boundedness. In fact, for any $(\phi(t), \varphi(t), \gamma(t)) \in H_{L}^{m}$, by (3.15) we have $\left(\widehat{z}_{1}(t), \widehat{z}_{2}(t), \widehat{z}_{3}(t)\right)=A(\phi(t), \varphi(t), \gamma(t)) \in H_{L}^{m}$; that is, it satisfies

$$
0<(m, m, m) \leq A(\phi(t), \varphi(t), \gamma(t))=\left(\widehat{z}_{1}(t), \widehat{z}_{2}(t), \widehat{z}_{3}(t)\right) \leq(L, L, L) .
$$

Next we prove equicontinuous. For any $(\phi(t), \varphi(t), \gamma(t)) \in H_{L}^{m}$, we denote $\left(\widehat{z}_{1}(t), \widehat{z}_{2}(t)\right.$, $\left.\widehat{z}_{3}(t)\right)=A(\phi(t), \varphi(t), \gamma(t)), t_{1}<t_{2}$, and then

$$
\begin{aligned}
\left|\widehat{z}_{1}\left(t_{1}\right)-\widehat{z}_{1}\left(t_{2}\right)\right|=\mid & \mid \int_{-\infty}^{t_{1}} e^{-\int_{s}^{t_{1}} a_{1}(r) d r}\left[b_{1}(s)+c_{1}(s) \frac{\phi(s)}{\varphi(s)}+d_{1}(s) \frac{\phi(s)}{\gamma(s)}-f_{1}(s) \phi^{2}(s)\right] \\
& -\int_{-\infty}^{t_{2}} e^{-\int_{s}^{t_{2}} a_{1}(r) d r}\left[b_{1}(s)+c_{1}(s) \frac{\phi(s)}{\varphi(s)}+d_{1}(s) \frac{\phi(s)}{r(s)}-f_{1}(s) \phi^{2}(s)\right] \mid
\end{aligned}
$$

Let $h_{1}(t)=b_{1}(t)+c_{1}(t)(\phi(t) / \varphi(t))+d_{1}(t)(\phi(t) / \gamma(t))-f_{1}(t) \phi^{2}(t)$; we obtain

$$
\begin{aligned}
\left|\widehat{z}_{1}\left(t_{2}\right)-\widehat{z}_{1}\left(t_{1}\right)\right| & =\left|\int_{-\infty}^{t_{2}} e^{-\int_{s}^{t_{2}} a_{1}(r) d r} h_{1}(s) d s-\int_{-\infty}^{t_{1}} e^{-\int_{s}^{t_{1}} a_{1}(r) d r} h_{1}(s) d s\right| \\
& \leq\left|\int_{t_{1}}^{t_{2}} e^{-\int_{s}^{t_{2}} a_{1}(r) d r} h_{1}(s) d s\right|+\left|\int_{-\infty}^{t_{1}} e^{-\int_{s}^{t_{1}} a_{1}(r) d r}\left(e^{-\int_{t_{1}}^{t_{2}} a_{1}(r) d r}-1\right) h_{1}(s) d s\right|
\end{aligned}
$$

Recalling $(\phi(t), \varphi(t), \gamma(t)) \in H_{L}^{m}$, we deduce that there exists a positive number $M$ such that $\left|h_{1}(s)\right| \leq M$; then (3.18) becomes

$$
\left|\widehat{z}_{1}\left(t_{2}\right)-\widehat{z}_{1}\left(t_{1}\right)\right| \leq M e^{-\int_{\xi_{1}}^{t_{2}} a_{1}(r) d r}\left|t_{2}-t_{1}\right|+\underset{\sim 1}{\frac{1}{a} M}\left|1-e^{-\int_{t_{1}}^{t_{2}} a_{1}(r) d r}\right|,
$$

where $\xi_{1} \in\left(t_{1}, t_{2}\right)$.

Similarly, we have

$$
\left|\widehat{z}_{2}\left(t_{2}\right)-\widehat{z}_{2}\left(t_{1}\right)\right| \leq N e^{-\int_{\xi_{2}}^{t_{2}} a_{2}(r) d r}\left|t_{1}-t_{2}\right|+\underset{\sim 2}{\frac{1}{a}} N\left|1-e^{-\int_{t_{1}}^{t_{2}} a_{2}(r) d r}\right|,
$$

where $\xi_{2} \in\left(t_{1}, t_{2}\right)$, and $N$ is a positive number.

By a completely analogous argument, we obtain

$$
\left|\widehat{z}_{3}\left(t_{2}\right)-\widehat{z}_{3}\left(t_{1}\right)\right| \leq P e^{-\int_{\xi_{3}}^{t_{2}} a_{3}(r) d r}\left|t_{1}-t_{2}\right|+\underset{\sim 3}{\frac{1}{a}} P\left|1-e^{-\int_{t_{1}}^{t_{2}} a_{3}(r) d r}\right|,
$$

where $\xi_{3} \in\left(t_{1}, t_{2}\right)$, and $P$ is a positive number. 
By (3.19)-(3.21), for any $(\phi(t), \varphi(t), \gamma(t)) \in H_{L}^{m}$, we derive

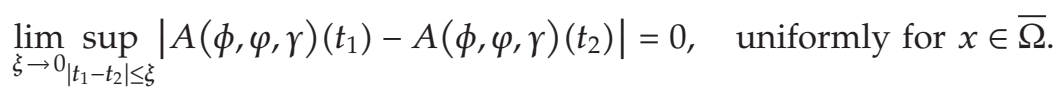

Thus, $A$ is a compact mapping which maps $H_{L}^{m}$ into itself; by Schauder fixed point theorem, there exists a fixed point $(\phi(t), \varphi(t), \gamma(t)) \in H_{L}^{m}$ for $A$; namely, (3.11) has a solution; therefore there exists a strictly positive almost periodic solution $\left(v_{1}^{*}(t), v_{2}^{*}(t), v_{3}^{*}(t)\right)=$ $(1 / \phi(t), 1 / \varphi(t), 1 / \gamma(t)), t \in R^{+}$for system (3.10). It is obvious that $\left(v_{1}^{*}(t), v_{2}^{*}(t), v_{3}^{*}(t)\right), t \in R^{+}$ is also the spatial homogeneity almost periodic solution for (1.1).

Theorem 3.2. Under the conditions of Theorem 3.1, suppose that system (1.1) satisfies the following conditions:

$$
\begin{gathered}
\sup _{t \geq 0}\left(b_{3}(t)+b_{2}(t)-b_{1}(t)\right)=-\varepsilon_{1}<0, \quad \sup _{t \geq 0}\left(c_{3}(t)+c_{1}(t)-c_{2}(t)\right)=-\varepsilon_{2}<0, \\
\sup _{t \geq 0}\left(d_{1}(t)+d_{2}(t)-d_{3}(t)\right)=-\varepsilon_{3}<0 .
\end{gathered}
$$

Then there exists a strictly positive spatial homogeneity almost periodic solution $\left(v_{1}^{*}(t), v_{2}^{*}(t), v_{3}^{*}(t)\right)$ for (1.1), and the corresponding solution for systems (1.1)-(1.3) is globally asymptotically stable; that is, the solution $\left(v_{1}(x, t), v_{2}(x, t), v_{3}(x, t)\right),(x, t) \in \bar{\Omega} \times R^{+}$satisfies

$$
\lim _{t \rightarrow \infty}\left(v_{i}(x, t)-v_{i}^{*}(t)\right)=0, \quad i=1,2,3, \text { uniformly for } x \in \bar{\Omega}
$$

Proof. We have obtained the existence by Theorem 3.1; next we pay more attention to the stability. Concerning (3.24), we have two cases on initial data $v_{i 0}(x), i=1,2,3$.

(1) $v_{i 0}(x)>0, x \in \bar{\Omega}$.

(2) There exists a point $x_{0} \in \bar{\Omega}$, such that $v_{10}\left(x_{0}\right)=0, v_{20}\left(x_{0}\right)=0$ or $v_{30}\left(x_{0}\right)=0$.

For the case (1), let $l_{i}=\min _{x \in \bar{\Omega}} v_{i 0}(x), r_{i}=\max _{x \in \bar{\Omega}} v_{i 0}(x), i=1,2,3$; then $0<l_{i} \leq$ $v_{i 0}(x) \leq r_{i}$. Suppose that $\left(\bar{v}_{1}(t), \bar{v}_{2}(t), \bar{v}_{3}(t)\right)$ and $\left(\underline{v}_{1}(t), \underline{v}_{2}(t), \underline{v}_{3}(t)\right)$ are the solution for (3.10) corresponding to initial datum $\left(\bar{v}_{1}(0), \bar{v}_{2}(0), \bar{v}_{3}(0)\right)=\left(r_{1}, r_{2}, r_{3}\right)$ and $\left(\underline{v}_{1}(0), \underline{v}_{2}(0), \underline{v}_{3}(0)\right)=$ $\left(l_{1}, l_{2}, l_{3}\right)$, respectively; then there are a pair of ordered upper and lower solutions $\left(\bar{v}_{1}(t), \bar{v}_{2}(t), \bar{v}_{3}(t)\right)$ and $\left(\underline{v}_{1}(t), \underline{v}_{2}(t), \underline{v}_{3}(t)\right)$ for $(1.1)-(1.3)$; by Lemma 2.5 , there exists a unique solution $\left(v_{1}(x, t), v_{2}(x, t), v_{3}(x, t)\right),(x, t) \in \bar{\Omega} \times R^{+}$for system (1.1)-(1.3), which satisfies

$$
\left(\underline{v}_{1}(t), \underline{v}_{2}(t), \underline{v}_{3}(t)\right) \leq\left(v_{1}(x, t), v_{2}(x, t), v_{3}(x, t)\right) \leq\left(\bar{v}_{1}(t), \bar{v}_{2}(t), \bar{v}_{3}(t)\right)
$$

If we have

$$
\lim _{t \rightarrow \infty}\left[\bar{v}_{i}(t)-v_{i}^{*}(t)\right]=\lim _{t \rightarrow \infty}\left[\underline{v}_{i}(t)-v_{i}^{*}(t)\right]=0, \quad i=1,2,3,
$$


then (3.24) holds. Therefore, if we want to obtain (3.26), we only need to prove that the solution $\left(v_{1}(t), v_{2}(t), v_{3}(t)\right)$ for (3.10) with arbitrary positive initial data $\left(v_{1}(0), v_{2}(0), v_{3}(0)\right)=$ $\left(v_{10}, v_{20}, v_{30}\right)$ satisfies

$$
\lim _{t \rightarrow \infty}\left(v_{i}(t)-v_{i}^{*}(t)\right)=0, \quad i=1,2,3
$$

Because of the initial datum $\left(v_{10}, v_{20}, v_{30}\right)>0$ and grazing rates $\left.\underset{\sim 1}{f} \underset{\sim}{f} \underset{\sim}{f} \underset{\sim 3}{f}\right)>0$, by the practical meaning in biology, we know that $\left(v_{1}(t), v_{2}(t), v_{3}(t)\right)>0$. Now let

$$
P_{i}(t)=\ln v_{i}(t), \quad Q_{i}(t)=\ln v_{i}^{*}(t), \quad i=1,2,3 .
$$

Then one has

$$
\begin{aligned}
\frac{d}{d t}\left(P_{1}(t)-Q_{1}(t)\right)= & -b_{1}(t)\left(e^{P_{1}(t)}-e^{Q_{1}(t)}\right)-c_{1}(t)\left(e^{P_{2}(t)}-e^{Q_{2}(t)}\right)-d_{1}(t)\left(e^{P_{3}(t)}-e^{Q_{3}(t)}\right) \\
& +\left(\frac{1}{v_{1}(t)}-\frac{1}{v_{1}^{*}(t)}\right) f_{1}(t), \\
\frac{d}{d t}\left(P_{2}(t)-Q_{2}(t)\right)= & -b_{2}(t)\left(e^{P_{1}(t)}-e^{Q_{1}(t)}\right)-c_{2}(t)\left(e^{P_{2}(t)}-e^{Q_{2}(t)}\right)-d_{2}(t)\left(e^{P_{3}(t)}-e^{Q_{3}(t)}\right) \\
& +\left(\frac{1}{v_{2}(t)}-\frac{1}{v_{2}^{*}(t)}\right) f_{2}(t), \\
\frac{d}{d t}\left(P_{3}(t)-Q_{3}(t)\right)= & -b_{3}(t)\left(e^{P_{1}(t)}-e^{Q_{1}(t)}\right)-c_{3}(t)\left(e^{P_{2}(t)}-e^{Q_{2}(t)}\right)-d_{3}(t)\left(e^{P_{3}(t)}-e^{Q_{3}(t)}\right) \\
& +\left(\frac{1}{v_{3}(t)}-\frac{1}{v_{3}^{*}(t)}\right) f_{3}(t) .
\end{aligned}
$$

Namely,

$$
\begin{aligned}
\frac{d}{d t}\left(P_{1}(t)-Q_{1}(t)\right)= & -\left(b_{1}(t)+\frac{f_{1}(t)}{v_{1}(t) v_{1}^{*}(t)}\right)\left(e^{P_{1}(t)}-e^{Q_{1}(t)}\right)-c_{1}(t)\left(e^{P_{2}(t)}-e^{Q_{2}(t)}\right) \\
& -d_{1}(t)\left(e^{P_{3}(t)}-e^{Q_{3}(t)}\right) \\
\frac{d}{d t}\left(P_{2}(t)-Q_{2}(t)\right)= & -b_{2}(t)\left(e^{P_{1}(t)}-e^{Q_{1}(t)}\right)-\left(c_{2}(t)+\frac{f_{2}(t)}{v_{2}(t) v_{2}^{*}(t)}\right)\left(e^{P_{2}(t)}-e^{Q_{2}(t)}\right) \\
& -d_{2}(t)\left(e^{P_{3}(t)}-e^{Q_{3}(t)}\right), \\
\frac{d}{d t}\left(P_{3}(t)-Q_{3}(t)\right)= & -b_{3}(t)\left(e^{P_{1}(t)}-e^{Q_{1}(t)}\right)-c_{3}(t)\left(e^{P_{2}(t)}-e^{Q_{2}(t)}\right) \\
& -\left(d_{3}(t)+\frac{f_{3}(t)}{v_{3}(t) v_{3}^{*}(t)}\right)\left(e^{P_{3}(t)}-e^{Q_{3}(t)}\right) .
\end{aligned}
$$


Consider the following Lyapunov function:

$$
U(t)=\sum_{i=1}^{3}\left|P_{i}(t)-Q_{i}(t)\right|, \quad t \geq 0
$$

Let $D^{+} U$ represent the right derivation on function $U(t)$; we have

$$
\begin{aligned}
D^{+} U(t) & \\
= & \sum_{i=1}^{3} D^{+}\left|P_{i}(t)-Q_{i}(t)\right|=\sum_{i=1}^{3} \operatorname{sgn}\left(P_{i}(t)-Q_{i}(t)\right) \frac{d}{d t}\left(P_{i}(t)-Q_{i}(t)\right) \\
= & \operatorname{sgn}\left(P_{1}(t)-Q_{1}(t)\right) \\
& \times\left[-\left(b_{1}(t)+\frac{f_{1}(t)}{v_{1}(t) v_{1}^{*}(t)}\right)\left(e^{P_{1}(t)}-e^{Q_{1}(t)}\right)-c_{1}(t)\left(e^{P_{2}(t)}-e^{Q_{2}(t)}\right)-d_{1}(t)\left(e^{P_{3}(t)}-e^{Q_{3}(t)}\right)\right] \\
& +\operatorname{sgn}\left(P_{2}(t)-Q_{2}(t)\right) \\
& \times\left[-b_{2}(t)\left(e^{P_{1}(t)}-e^{Q_{1}(t)}\right)-\left(c_{2}(t)+\frac{f_{2}(t)}{v_{2}(t) v_{2}^{*}(t)}\right)\left(e^{P_{2}(t)}-e^{Q_{2}(t)}\right)-d_{2}(t)\left(e^{P_{3}(t)}-e^{Q_{3}(t)}\right)\right] \\
& +\operatorname{sgn}\left(P_{3}(t)-Q_{3}(t)\right) \\
& \times \\
& {\left[-b_{3}(t)\left(e^{P_{1}(t)}-e^{Q_{1}(t)}\right)-c_{3}(t)\left(e^{P_{2}(t)}-e^{Q_{2}(t)}\right)-\left(d_{3}(t)+\frac{f_{3}(t)}{v_{3}(t) v_{3}^{*}(t)}\right)\left(e^{P_{3}(t)}-e^{Q_{3}(t)}\right)\right] } \\
\leq & \left(b_{3}(t)+b_{2}(t)-b_{1}(t)\right)\left|e^{P_{1}(t)}-e^{Q_{1}(t)}\right|+\left(c_{1}(t)+c_{3}(t)-c_{2}(t)\right)\left|e^{P_{2}(t)}-e^{Q_{2}(t)}\right| \\
& +\left(d_{1}(t)+d_{2}(t)-d_{3}(t)\right)\left|e^{P_{3}(t)}-e^{Q_{3}(t)}\right| \\
\leq & -\varepsilon_{1}\left|v_{1}(t)-v_{1}^{*}(t)\right|-\varepsilon_{2}\left|v_{2}(t)-v_{2}^{*}(t)\right|-\varepsilon_{3}\left|v_{3}(t)-v_{3}^{*}(t)\right| .
\end{aligned}
$$

Integrated by the time, we have

$$
U(t)+\sum_{i=1}^{3} \varepsilon_{i} \int_{0}^{t}\left|v_{i}(s)-v_{i}^{*}(s)\right| d s \leq U(0) .
$$

By the nonnegative of $U(t)$ and the boundedness of $U(0)$, we obtain that the $U(t)$ is bounded, and

$$
\int_{0}^{t}\left|v_{i}(t)-v_{i}^{*}(t)\right| d s, \quad i=1,2,3
$$

convergences, by (3.32) we get $D^{+} U(t)<0$, then the limit

$$
\lim _{t \rightarrow \infty} U(t)=l
$$


exists, and $U(t) \geq l$. If $l>0$, then at least one of the following three inequalities

$$
\left|P_{1}(t)-Q_{1}(t)\right|>\frac{l}{4}, \quad\left|P_{2}(t)-Q_{2}(t)\right|>\frac{l}{4}, \quad\left|P_{3}(t)-Q_{3}(t)\right|>\frac{l}{4}
$$

holds. Without loss of generality, we assume that $\left|P_{1}(t)-Q_{1}(t)\right|>l / 4$. Thus there is no point of intersection between $P_{1}(t)$ and $Q_{1}(t)$. Suppose that $P_{1}(t)>Q_{1}(t)$; then we have $P_{1}(t)-Q_{1}(t)>$ $l / 4$. Thus

$$
\begin{aligned}
\int_{0}^{t}\left|v_{1}(t)-v_{1}^{*}(t)\right| d s & =\int_{0}^{t}\left|e^{P_{1}(s)}-e^{Q_{1}(s)}\right| d s=\int_{0}^{t} e^{Q_{1}(s)}\left|e^{P_{1}(s)-Q_{1}(s)}-1\right| d s \\
& \geq m \int_{0}^{t}\left(e^{P_{1}(s)-Q_{1}(s)}-1\right) d s>m \int_{0}^{t}\left(e^{l / 4}-1\right) d s=m\left(e^{l / 4}-1\right) t \longrightarrow+\infty,
\end{aligned}
$$

which contradicts with the convergence of $\int_{0}^{t}\left|v_{i}(s)-v_{i}^{*}(s)\right| d s$. Therefore $l=0$; consequently

$$
\lim _{t \rightarrow \infty}\left|v_{i}(t)-v_{i}^{*}(t)\right|=0, \quad i=1,2,3
$$

Then we obtain (3.27). such that

For the case (2), firstly, choose three sufficient large positive numbers $M_{1}, M_{2}$, and $M_{3}$,

$$
\begin{array}{ll}
f_{1}(t) \leq-M_{1}\left(a_{1}(t)-b_{1}(t) M_{1}\right), & t>0, \\
f_{2}(t) \leq-M_{2}\left(a_{2}(t)-c_{2}(t) M_{2}\right), & t>0, \\
f_{3}(t) \leq-M_{3}\left(a_{3}(t)-d_{3}(t) M_{3}\right), & t>0,
\end{array}
$$

and $M_{i} \geq \max _{x \in \bar{\Omega}} v_{i 0}(x), i=1,2,3$. Let $\underset{\sim i}{v}=0, \tilde{v}_{i}=M_{i}, i=1,2,3$; then we have

$$
\begin{aligned}
& \frac{\partial \widetilde{v}_{1}}{\partial t}-k_{1}(t) \Delta \widetilde{v}_{1}-\widetilde{v}_{1}\left[a_{1}(t)-b_{1}(t) \widetilde{v}_{1}-c_{1}(t) \underset{\sim 2}{v}-d_{1}(t) \underset{\sim 3}{v}\right]-f_{1}(t) \geq 0, \\
& \frac{\partial \underset{\sim}{v}}{\partial t}-k_{1}(t) \Delta \underset{\sim 1}{v}-\underset{\sim 1}{v}\left[a_{1}(t)-b_{1}(t) \underset{\sim 1}{\underset{v}{v}}-c_{1}(t) \widetilde{v}_{2}-d_{1}(t) \widetilde{v}_{3}\right]-f_{1}(t) \leq 0 \text {, } \\
& \frac{\partial \widetilde{v}_{2}}{\partial t}-k_{2}(t) \Delta \widetilde{v}_{2}-\widetilde{v}_{2}\left[a_{2}(t)-b_{2}(t) \underset{\sim 1}{v}-c_{2}(t) \tilde{v}_{2}-d_{2}(t) \underset{\sim 3}{v}\right]-f_{2}(t) \geq 0 \text {, } \\
& \frac{\partial v}{\partial t}-k_{2}(t) \Delta \underset{\sim 2}{v}-\underset{\sim 2}{v}\left[a_{2}(t)-b_{2}(t) \widetilde{v}_{1}-c_{2}(t) \underset{\sim 2}{\underset{v}{v}}-d_{2}(t) \widetilde{v}_{3}\right]-f_{2}(t) \leq 0 \text {, } \\
& \frac{\partial \widetilde{v}_{3}}{\partial t}-k_{3}(t) \Delta \widetilde{v}_{3}-\widetilde{v}_{3}\left[a_{3}(t)-b_{3}(t) \underset{\sim 1}{v}-c_{3}(t) \underset{\sim 2}{v}-d_{3}(t) \widetilde{v}_{3}\right]-f_{3}(t) \geq 0 \text {, } \\
& \frac{\partial v}{\partial t}-k_{3}(t) \Delta \underset{\sim 3}{v}-\underset{\sim_{3}}{v}\left[a_{3}(t)-b_{3}(t) \tilde{v}_{1}-c_{3}(t) \tilde{v}_{2}-d_{3}(t) \underset{\sim_{3}}{\underset{v}{v}}\right]-f_{3}(t) \leq 0 .
\end{aligned}
$$


Namely, $\underset{\sim i}{v}=0$, and $\tilde{v}_{i}=M_{i}, i=1,2,3$ are a pair of ordered upper and lower solutions for systems $\underset{\sim}{(1.1)}-(1.3)$. By Lemma 2.5 , there exists a unique solution $\left(v_{1}(x, t), v_{2}(x, t), v_{3}(x, t)\right)$ for systems (1.1)-(1.3), which satisfy

$$
0 \leq v_{i}(x, t) \leq M_{i}, \quad i=1,2,3 ;(x, t) \in \bar{\Omega} \times[0, \infty) .
$$

Secondly, we choose positive numbers $\delta_{1}, \delta_{2}$, and $\delta_{3}$ such that

$$
\begin{array}{ll}
\delta_{1}+a_{1}(t)-b_{1}(t) v_{1}(x, t)-c_{1}(t) v_{2}(x, t)-d_{1}(t) v_{3}(x, t)>0, & (x, t) \in \bar{\Omega} \times[0, \infty), \\
\delta_{2}+a_{2}(t)-b_{2}(t) v_{1}(x, t)-c_{2}(t) v_{2}(x, t)-d_{2}(t) v_{3}(x, t)>0, & (x, t) \in \bar{\Omega} \times[0, \infty), \\
\delta_{3}+a_{3}(t)-b_{3}(t) v_{1}(x, t)-c_{3}(t) v_{2}(x, t)-d_{3}(t) v_{3}(x, t)>0, & (x, t) \in \bar{\Omega} \times[0, \infty) .
\end{array}
$$

Accordingly, we have

$$
\begin{aligned}
& \frac{\partial v_{1}}{\partial t}-k_{1}(t) \Delta v_{1}+\delta_{1} v_{1}=v_{1}\left[\delta_{1}+a_{1}(t)-b_{1}(t) v_{1}-c_{1}(t) v_{2}-d_{1}(t) v_{3}\right]+f_{1}(t) \geq 0 \\
& \frac{\partial v_{2}}{\partial t}-k_{2}(t) \Delta v_{2}+\delta_{2} v_{2}=v_{2}\left[\delta_{2}+a_{2}-b_{2}(t) v_{1}-c_{2}(t) v_{2}-d_{2}(t) v_{3}\right]+f_{2}(t) \geq 0 \\
& \frac{\partial v_{3}}{\partial t}-k_{3}(t) \Delta v_{3}+\delta_{3} v_{3}=v_{3}\left[\delta_{3}+a_{3}-b_{3}(t) v_{1}-c_{3}(t) v_{2}-d_{3}(t) v_{3}\right]+f_{3}(t) \geq 0
\end{aligned}
$$

Next, we prove $v_{i}(x, t)>0$ in $\bar{\Omega} \times(0, \infty)$ for $i=1,2,3$. Firstly, we show $v_{i}(x, t)>0$ in $\Omega \times(0, \infty)$. If there exists one point $\left(x_{0}, t_{0}\right) \in \Omega \times(0, \infty)$ such that $v_{i}\left(x_{0}, t_{0}\right)=0$, by extremum principle, we have $v_{i}(x, t) \equiv 0$ in $\bar{\Omega} \times\left[0, t_{0}\right)$. However $v_{i}(x, 0)=v_{i 0}(x) \geq 0$, and not being constant zero, we obtain a contradiction. Therefore we have $v_{i}(x, t)>0$ in $\Omega \times(0, \infty)$. Then we show $v_{i}(x, t)>0$ in $\partial \Omega \times(0, \infty)$. If there exists a point $\left(x_{0}, t_{0}\right) \in \partial \Omega \times(0, \infty)$ such that $v_{i}\left(x_{0}, t_{0}\right)=0$, by the extremum principle, we have $\partial v_{i}(x, t) / \partial n<0$, where $(x, t) \in \partial \Omega \times(0, \infty)$, which is contrary with boundary conditions (1.2). Thus we have $v_{i}(x, t)>0$ in $\bar{\Omega} \times(0, \infty)$.

For a fixed number $\lambda>0$, by (3.41), we have

$$
0<v_{i}(x, \lambda) \leq M_{i}, \quad i=1,2,3, x \in \bar{\Omega}
$$

Because $v_{i}(x, t+\lambda)$ satisfy system $(1.1)$ in $\bar{\Omega} \times(0, \infty)$ and the conditions $(1.2)$ in $\partial \Omega \times(0, \infty)$, thereby $\left(v_{1}(x, t+\lambda), v_{2}(x, t+\lambda), v_{3}(x, t+\lambda)\right)$ is regarded as a solution for system (1.1) under initial data $\left(\widehat{v}_{10}(x), \widehat{v}_{20}(x), \widehat{v}_{30}(x)\right)=\left(v_{1}(x, \lambda), v_{2}(x, \lambda), v_{3}(x, \lambda)\right)$, nevertheless, we have $\widehat{v}_{i 0}>0$ in $\bar{\Omega}, i=1,2,3$; combining the conclusions in case (1), we have

$$
\lim _{t \rightarrow \infty}\left(v_{i}(x, t+\lambda)-v_{i}^{*}(t)\right)=0, \quad i=1,2,3, \text { uniformly for } x \in \bar{\Omega}
$$

By the arbitrariness of $\lambda$, we obtain

$$
\lim _{t \rightarrow \infty}\left(v_{i}(x, t)-v_{i}^{*}(t)\right)=0, \quad i=1,2,3, \text { uniformly for } x \in \bar{\Omega} .
$$


If $k_{i}(t), a_{i}(t), b_{i}(t), c_{i}(t), d_{i}(t)$, and $f_{i}(t)(i=1,2,3)$ of (1.1) are periodic functions in real number field $R$, respectively, then we have the following results.

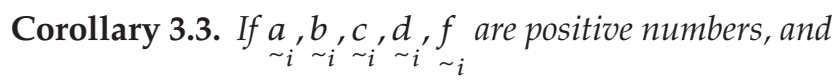

$$
\frac{\left(\tilde{b}_{i}+\tilde{c}_{i}+\tilde{d}_{i}\right)}{\underset{\sim i}{a}} \leq L=\min \left\{\sqrt{\frac{\underset{\sim}{b}}{\tilde{\tilde{f}}_{1}}}, \sqrt{\frac{\underset{\sim}{c}}{\tilde{f}_{2}}}, \sqrt{\frac{\underset{\sim}{d}}{\tilde{f}_{3}}}, \frac{(\underset{\sim 1}{d}+\underset{\sim 1}{c})}{\tilde{a}_{1}}, \frac{(\underset{\sim 2}{b}+\underset{\sim 2}{d})}{\tilde{a}_{2}}, \frac{\underset{\sim 3}{b}+\underset{\sim 3}{b})}{\tilde{a}_{3}}\right\}
$$

are satisfied for $i=1,2,3$, then there exists a strictly positive spatial homogeneity periodic solution $V(t)=\left(\widehat{v}_{1}(t), \widehat{v}_{2}(t), \widehat{v}_{3}(t)\right)$ for $(1.1)$.

Corollary 3.4. Under the conditions of Corollary 3.3, suppose that system (1.1) satisfies the following conditions:

$$
\begin{gathered}
\sup _{t \geq 0}\left(b_{3}(t)+b_{2}(t)-b_{1}(t)\right)=-\varepsilon_{1}<0, \quad \sup _{t \geq 0}\left(c_{3}(t)+c_{1}(t)-c_{2}(t)\right)=-\varepsilon_{2}<0, \\
\sup _{t \geq 0}\left(d_{1}(t)+d_{2}(t)-d_{3}(t)\right)=-\varepsilon_{3}<0 .
\end{gathered}
$$

Then there exists a strictly positive spatial homogeneity periodic solution $\left(v_{1}^{*}(t), v_{2}^{*}(t), v_{3}^{*}(t)\right)$ for $(1.1)$, and the corresponding solution for systems (1.1)-(1.3) is globally asymptotically stable; that is, the solution $\left(v_{1}(x, t), v_{2}(x, t), v_{3}(x, t)\right),(x, t) \in \bar{\Omega} \times R^{+}$satisfies

$$
\lim _{t \rightarrow \infty}\left(v_{i}(x, t)-v_{i}^{*}(t)\right)=0, \quad i=1,2,3, \text { uniformly for } x \in \bar{\Omega} .
$$

\section{Conclusion}

This paper presents the use of upper and lower solutions method for systems of nonlinear reaction-diffusion equations. This method is a powerful tool for solving nonlinear differential equations in mathematical physics, chemistry, and engineering, and so forth. The technique constructing a pair of upper and lower solutions and Lyapunov function provides a new efficient method to handle the nonlinear structure.

We have dealt with the problem of almost periodic solution for a three-species competition system with grazing rates and diffusions. The general sufficient conditions have been obtained to ensure the existence and stability of the strictly positive space homogenous almost periodic solution for the nonlinear reaction-diffusion equations. These criteria generalize and improve some known results. In particular, the sufficient conditions that we obtained are very simple, which provide flexibility for the application and analysis of nonlinear three-species competition system.

\section{Acknowledgments}

The authors are grateful to the reviewers for their comments which helped in improving the paper. This work is supported by the Science and Technology Project of Chongqing 
Municipal Education Committee (Grant no. kJ110501) of China, the NSFC (Grant nos. 51005264, 40801214) of China, and Natural Science Foundation Project of CQ CSTC (Grant no. 2010BB9401) of China.

\section{References}

[1] D. P. Jiang, "Some periodic ecological models with grazing rates," Journal of Biomathematics, vol. 5, no. 1, pp. 80-89, 1990 (Chinese).

[2] Q. C. Lin, "Some almost periodic biological models with grazing rates," Journal of Biomathematics, vol. 14, no. 3, pp. 257-263, 1999 (Chinese).

[3] F. D. Chen and X. X. Chen, "The n-competing Lotka-Volterra almost periodic systems with grazing rates," Journal of Biomathematics, vol. 18, no. 4, pp. 411-416, 2003 (Chinese).

[4] Z. Q. Zhang and Z. C. Wang, "Periodic solution for a two-species nonautonomous competition LotkaVolterra patch system with time delay," Journal of Mathematical Analysis and Applications, vol. 265, no. 1, pp. 38-48, 2002.

[5] Z. Q. Zhang and Z. C. Wang, "Multiple positive periodic solutions for a generalized delayed population model with an exploited term," Science in China Series A, vol. 50, no. 1, pp. 27-34, 2007.

[6] D. Hu and Z. Q. Zhang, "Four positive periodic solutions of a discrete time delayed predatorprey system with nonmonotonic functional response and harvesting," Computers $\mathcal{E}$ Mathematics with Applications, vol. 56, no. 12, pp. 3015-3022, 2008.

[7] C. V. Pao and Y.M. Wang, "Numerical solutions of a three-competition Lotka-Volterra system," Applied Mathematics and Computation, vol. 204, no. 1, pp. 423-440, 2008.

[8] Y. Q. Liu, S. L. Xie, and Z. D. Xie, "Existence and stability for periodic solution of competition reactiondiffusion models with grazing rates in population dynamics," Journal of Systems Science and Systems Engineering, vol. 10, no. 2, pp. 402-410, 1996.

[9] C. Y. Wang, S. Q. An, and C. J. Fang, "Almost periodic solutions and periodic solutions of reactiondiffusion systems with time delays," Mathematica Applicata, vol. 20, no. 2, pp. 281-285, 2007 (Chinese).

[10] F. D. Chen and C. L. Shi, "Global attractivity in an almost periodic multi-species nonlinear ecological model," Applied Mathematics and Computation, vol. 180, no. 1, pp. 376-392, 2006.

[11] C. Y. Wang, "Existence and stability of periodic solutions for parabolic systems with time delays," Journal of Mathematical Analysis and Applications, vol. 339, no. 2, pp. 1354-1361, 2008.

[12] C. Y. Wang, S. Wang, and L. R. Li, "Periodic solution and almost periodic solution for a nonmonotone reaction-diffusion system with time delay," Acta Mathematica Scientia Series A, vol. 30, no. 2, pp. 517524, 2010 (Chinese).

[13] X. Q. Liu, "Periodic or almost periodic solutions to a class of systems of reaction-diffusion equations," Chinese Journal of Engineering Mathematics, vol. 11, no. 4, pp. 107-111, 1994 (Chinese).

[14] Y. Li and Y. Kuang, "Periodic solutions of periodic delay Lotka-Volterra equations and systems," Journal of Mathematical Analysis and Applications, vol. 255, no. 1, pp. 260-280, 2001.

[15] C. Y. Wang and X. H. Hu, "Existence and uniqueness of bounded solution and periodic solution of reaction-diffusion equation with time delay," Journal of Chongqing University of Posts and Telecommunication (Natural Science Edition), vol. 17, no. 5, pp. 644-646, 2005 (Chinese).

[16] C. Y. Wang, "Periodic solution of prey predator model with diffusion and distributed delay effects," Journal of Chongqing University of Posts and Telecommunication (Natural Science Edition), vol. 18, no. 3, pp. 409-412, 2006.

[17] C. V. Pao, Nonlinear Parabolic and Elliptic Equations, Plenum Press, New York, NY,USA, 1992.

[18] A. M. Fink, Almost Periodic Differential Equations, vol. 377 of Lecture Notes in Mathematics, Springer, Berlin, Germany, 1974. 


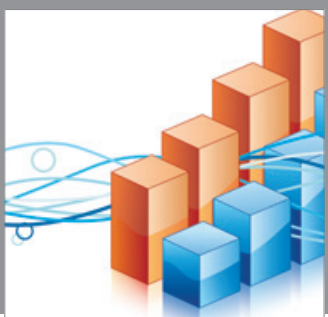

Advances in

Operations Research

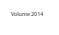

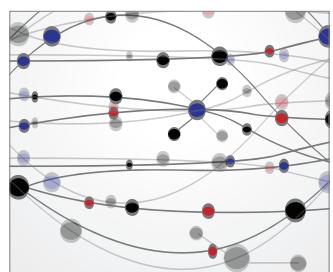

\section{The Scientific} World Journal
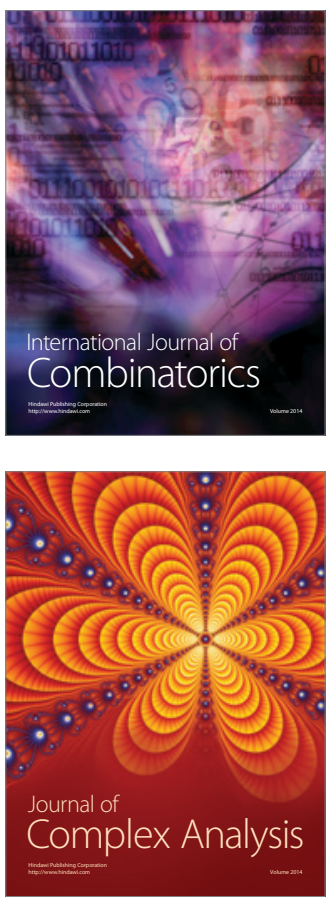

International Journal of

Mathematics and

Mathematical

Sciences
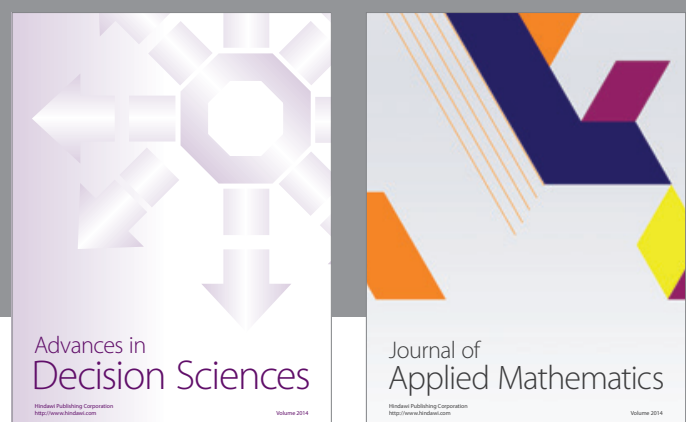

Journal of

Applied Mathematics
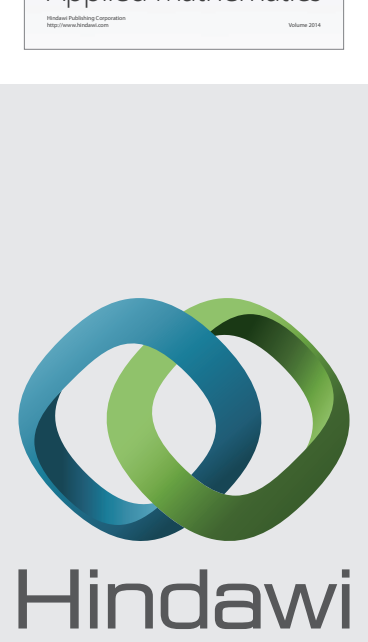

Submit your manuscripts at http://www.hindawi.com
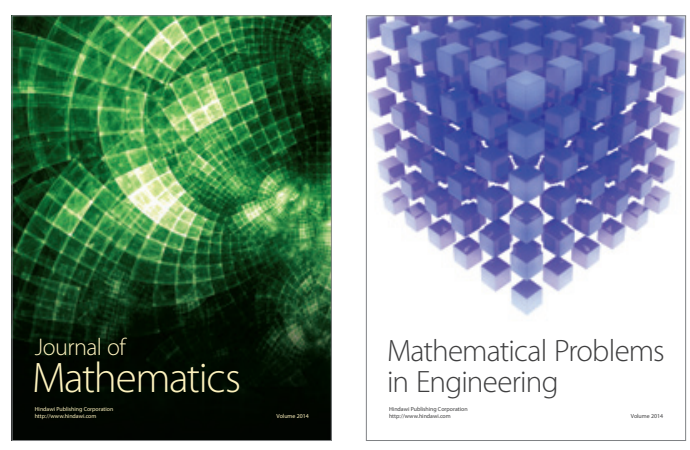

Mathematical Problems in Engineering
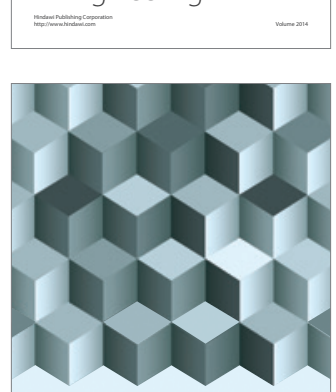

Journal of

Function Spaces
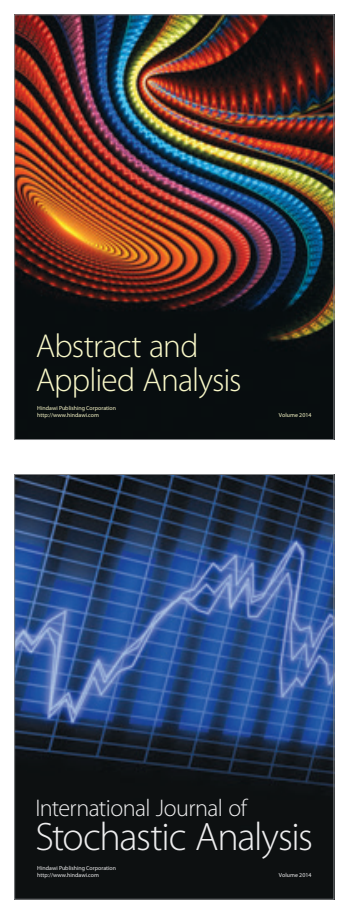

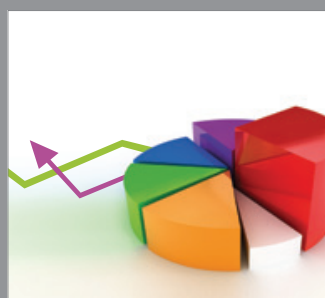

ournal of

Probability and Statistics

Promensencen
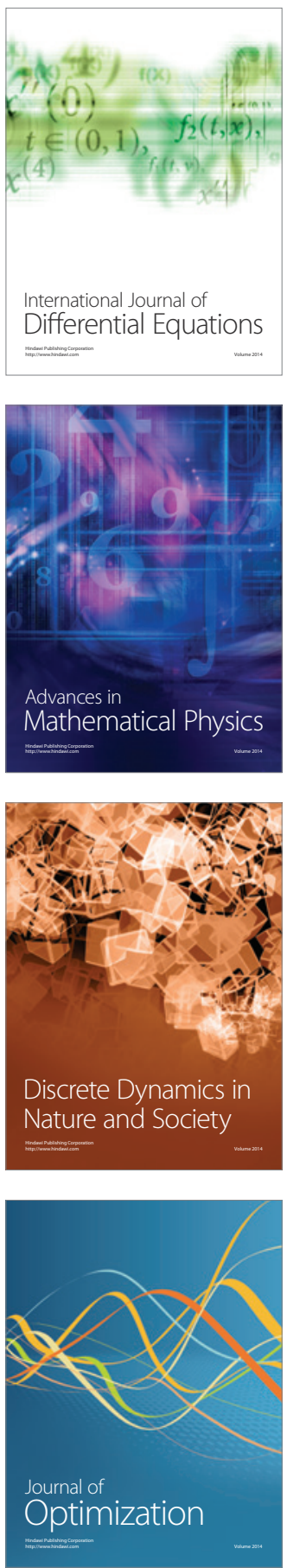